\title{
GONADOTROPINS IN OVULATION INDUCTION
}

\author{
Promodita Saravanan ${ }^{1}$, Nidhi Sharma ${ }^{2}$
}

${ }_{1}^{1}$ MBBS Student, Saveetha Medical College, Saveetha University, Chennai, Tamilnadu, India.

2Professor, Department of Reproductive Medicine, ARC, Saveetha Medical College, Saveetha University, Chennai, India

\section{BACKGROUND}

ABSTRACT

Urinary and recombinant synthetic gonadotropin analogues have been useful in a variety of clinical scenarios in reproductive medicine. They can be used as substitution therapy, stimulation therapy, regulation therapy and hyper stimulation therapy. Gonadotropin analogues are used as substitution therapy for endogenous gonadotropins in patients with Group I hypothalamic pituitary failure. Gonadotropin analogues are also used as stimulation therapy in patients with hypothalamic pituitary dysfunction. Gonadotropins can also be used as regulation therapy when the ratio of endogenous gonadotropins is altered as in women with polycystic ovarian syndrome. In in-vitro fertilization cycles, gonadotropin analogues are extremely useful for controlled ovarian hyper-stimulation therapy to increase the yield of oocytes.

The aim of this review is to discuss the physiological rationale of gonadotropin use in controlled ovarian hyper stimulation. The factors that affect the choice and dose of gonadotropins in controlled ovarian stimulation are listed. The availability and efficacy of urinary and recombinant products are also discussed.

\section{KEY WORDS}

Gonadotropins, Follicle Stimulating Hormone, Luteinizing Hormone, Recombinant, Urinary, and Human Chorionic Gonadotropin. HOW TO CITE THIS ARTICLE: Saravanan P, Sharma N. Gonadotropins in ovulation induction. J. Evolution Med. Dent. Sci. 2019;8(18):1498-1502, DOI: 10.14260/jemds/2019/333

\section{BACKGROUND}

There are two gonadotropins and two cells that are required for estradiol synthesis. This has been emphasized as the Sir Leon Speroff's "two cell, two gonadotropin theory."(1) Luteinizing hormone acts on theca cell that synthesizes androgens and Follicle stimulating hormone acts on granulosa cell for final aromatization of theca cell derived androgens to oestrogens. In IVF cycles, patient's endogenous pituitary is down regulated by GnRH agonist protocol or $\mathrm{GnRh}$ antagonist protocol and exogenous gonadotropins are injected.

The different indications are Clomiphene citrate failure, Controlled Ovarian Hyper stimulation in ART, Hypothalamus pituitary insufficiency, Hypothalamus pituitary dysfunction with associated Hyperandrogenism, Hypothalamic dysfunction with normal prolactin \& normal Androgens and Premature luteinisation of follicles

The aim of Controlled ovarian hyper stimulation is adequate oocyte yield. An appropriate response is defined as retrieval of 8-10 oocytes as this should result in sufficient high quality embryos being available for transfer.(2) In centers where cryopreservation is available the retrieval of 12-15 oocytes is considered optimum as this allows for multiple attempts of embryo transfer and thereby contributing to higher cumulative pregnancy rates.(3,4) Abnormal response in controlled ovarian hyper stimulation is defined as retrieval of less than 5 or more than 20 oocytes. If less than five oocytes are harvested cycle cancellation chance is high and if there are more than 20 oocytes there is high risk of ovarian hyper stimulation syndrome.(5)

'Financial or Other Competing Interest': None.

Submission 07-03-2019, Peer Review 20-04-2019,

Acceptance 26-04-2019, Published 06-05-2019.

Corresponding Author:

Dr. Nidhi Sharma,

No. 5, Jayanthi Street, Dr. Seethapathi Nagar,

Velacherry, Chennai, Tamilnadu, India.

E-mail:drbonuramkumar@yahoo.co.in

DOI: $10.14260 /$ jemds $/ 2019 / 333$
The basic principle of ovulation induction in GnRh agonist protocol include the concepts of GnRh a - flare-up followed by down regulation, recruitment, trajectory of growth, selection \& dominance, atresia, FSH initially \& FSH + LH later. In a GnRh antagonist Protocol there is GnRh antagonist down regulation, recruitment, trajectory of growth, selection \& dominance, atresia, FSH initially and FSH + LH later.

\section{Folliculogenesis}

FSH controls follicular recruitment and growth. The granulosa cells in the recruited follicles secrete oestrogen. There is rise in oestrogen as follicle grows to 150-250 $\mathrm{pg} / \mathrm{ml} /$ follicle of $>15 \mathrm{~mm}$ diameter. The ovarian micro milieu becomes estrogenic. Rising estradiol level in the midfollicular phase induces the LH surge. There are 2 critical features that are required for endogenous LH surge. The first is the concentration of estradiol and second is the length of time during which the estradiol elevation is sustained. In ART cycles endogenous LH surge does not take place as the pituitary is down regulated and so supplementation of LH in later part of follicular phase is essential.

\section{FSH threshold and Window}

Selective rise in serum FSH beyond a critical threshold level during luteal - follicular transition is a potent stimulus for follicle recruitment. Follicle with lowest FSH threshold becomes the first in cohort to secrete Oestrogen. It is not only the increase in FSH but maintenance of this increase for a critical duration of time that is essential in rescuing a cohort of follicles from atresia [Figure 1 (a)]. It is believed that the cohort size of healthy early antral follicles recruited during the luteo-follicular transition is around ten per ovary.

\section{Luteinizing Hormone Threshold, Window and Ceiling}

LH receptors are constitutively present on theca cells. FSH induces LH receptors on granulosa cells. In presence of minimal LH (LH threshold), FSH is able to activate paracrine signalling (IGF \& Inhibin) and this sustains the thecal androgen synthesis. LH is required for complete maturation 
of follicle, oocyte competence and for supporting corpus luteum. LH unlike FSH is inhibitory at high doses. LH threshold is the amount of LH required for normal follicle and oocyte development. However, in the presence of excessive amount, LH adversely affects preovulatory follicles depending on stage of development ( $\mathrm{LH}$ ceiling). The concepts of LH threshold, LH window and LH ceiling are elaborated in Figure 1(b).

\section{Initial Patient Evaluation}

Before any intervention is initiated, preconceptional counseling should be provided emphasizing the importance of lifestyle, especially weight reduction and exercise in overweight women, smoking, and alcohol consumption. A detailed checklist helps to identify any hypothalamic, pituitary and ovarian disorders. This includes the evaluation of health \& fertility status. It is done by baseline transvaginal scan on Day two. Serum levels of D2 FSH, LH, and Estradiol are measured. Patient is also examined and investigated to rule out thyroid dysfunction, hyperprolactinemia, other endocrinopathies, intracranial tumours \&ovarian tumours.

The patient should also be counselled regarding high rate of multifetal gestation, ovarian hyper stimulation syndrome and ectopic pregnancy. Factors pertaining to cost and time commitment should be explained.

\section{Gonadotropin Preparations}

Analogues of Human pituitary gonadotropins were first commercially synthesized from menopausal urine of Nuns in convents of Austria. This had containing high concentrations of LH and FSH. The preparations are HMG (75 IU FSH \& 75 IU LH) E.g. Pergonal/Menogon, highly purified HMG $(<5 \%$ urinary proteins), Purified urinary FSH $(<0.7 \mathrm{IU}$ LH $)$ E.g. Metrodin. Highly purified urinary FSH $(<0.01$ IU LH $\&<5 \%$ urinary proteins) E.g. Metrodin HP.

Human Chorionic Gonadotropin has a molecular structural similarity with LH (Pregnyl/Profassi) and has been used as trigger for final maturation of oocytes. It is important to realize that in patients with hypo gonadotropic hypogonadism when the endogenous LH is not produced, it is prudent to use only HCG trigger and not GnRH agonist trigger as the patients pituitary is unresponsive to exogenous and endogenous GnRH agonist and flare response leading to $\mathrm{LH}$ surge does not happen after $\mathrm{GnRH}$ agonist trigger. The various recombinant preparations that are available are recombinant FSH E.g. Gonal F and Puregon, recombinant LH E.g. Luveris and recombinant HCG E.g. Ovitrelle.

The advantages of recombinant gonadotropins are no protein contaminants (prion protein found in urinary preparations), high specificity, and unlimited supply with batch-to-batch consistency, high purity and absence of contamination by other gonadotropins.(6) In some recent studies rFSH was found to more efficacious than highly purified urinary products.(7) Clinical pregnancy rate is significantly higher and more oocyte of good quality are harvested.(8) It was also found that lower doses gonadotropins are used for shorter treatment period to achieve adequate response. Recombinant FSH has lower threshold dose, high activity and in vivo bio potency. $(9,10)$

\section{Variables Affecting Response to Gonadotropins}

Clinical, biochemical and biophysical markers can predict the response to gonadotropins. Clinical parameters are age and Body mass Index.(11) Biochemical parameters are serum hormone levels of FSH, estradiol, inhibin B and antimullerian hormone.(12,13) Biophysical markers include antral follicle count, ovarian volume and ovarian stromal blood flow.(14)

\section{Chronological Age and Body Mass Index}

Chronological age is common parameter in studies on ovarian response.(15,16) However there may be differences in ovarian aging depending on ethnicity and this cannot be accepted as the single best indicator due to wide ranges of age of the onset of menopause.(16) It has been proved that adipose tissue is a source of estrone, and this may correlate to the dose of gonadotropins required.(17) Obesity is also associated with increased insulin levels that in turn decrease the Sex hormone binding globulin (SHBG) levels and increases the insulin like growth factor 1 (IGF-1) levels. Increased levels of IGF-1 in turn stimulate 5-alpha reductase activity. Decreased SHBG levels and increased 5-alpha reductase activity increases the testosterone levels.

\section{Basal Day 2 FSH}

Basal FSH is the assay most often used as a screening test for ovarian reserve however its predictive accuracy is limited due to intercycle variability in the same patient. A study has revealed that elevated Basal FSH reflects a quantitative rather a qualitative decline in ovarian reserve.(18) $\mathrm{A}$ high Basal FSH is not necessarily a contraindication of ovarian stimulation. A high basal FSH can possible correlate with gonadotropin dose.

\section{Antral Follicular Count}

There is a clear correlation between the number of antral follicular count seen at the beginning of the follicular phase during a natural cycle and the ovarian response.(19) These are the potentials of the ovary that can be stimulated and harvested.(20) However many analysis revealed that Antral follicular count reflected follicular output and not gonadotropin dose requirement.(21)

\section{Scoring Systems Developed to Calculate Gonadotropin Dose}

In some countries, a scoring model based on four parameters, antral follicular count, total Doppler score, serum testosterone and smoking habit has been developed.(22) Scoring systems are not adopted widely in clinical practice because a number of clinics do not measure total Doppler score and serum testosterone routinely. In addition, smoking is not widely prevalent in all communities.

Another scoring system to calculate the dose of gonadotropins is based on serum basal FSH, BMI, age and antral follicle count. These four factors were modelled into an algorithm to calculate the dose of recombinant FSH.(23) In this model, there were high cancellation rates due to the application of low starting dose of 75 IU for multiple follicular development. 


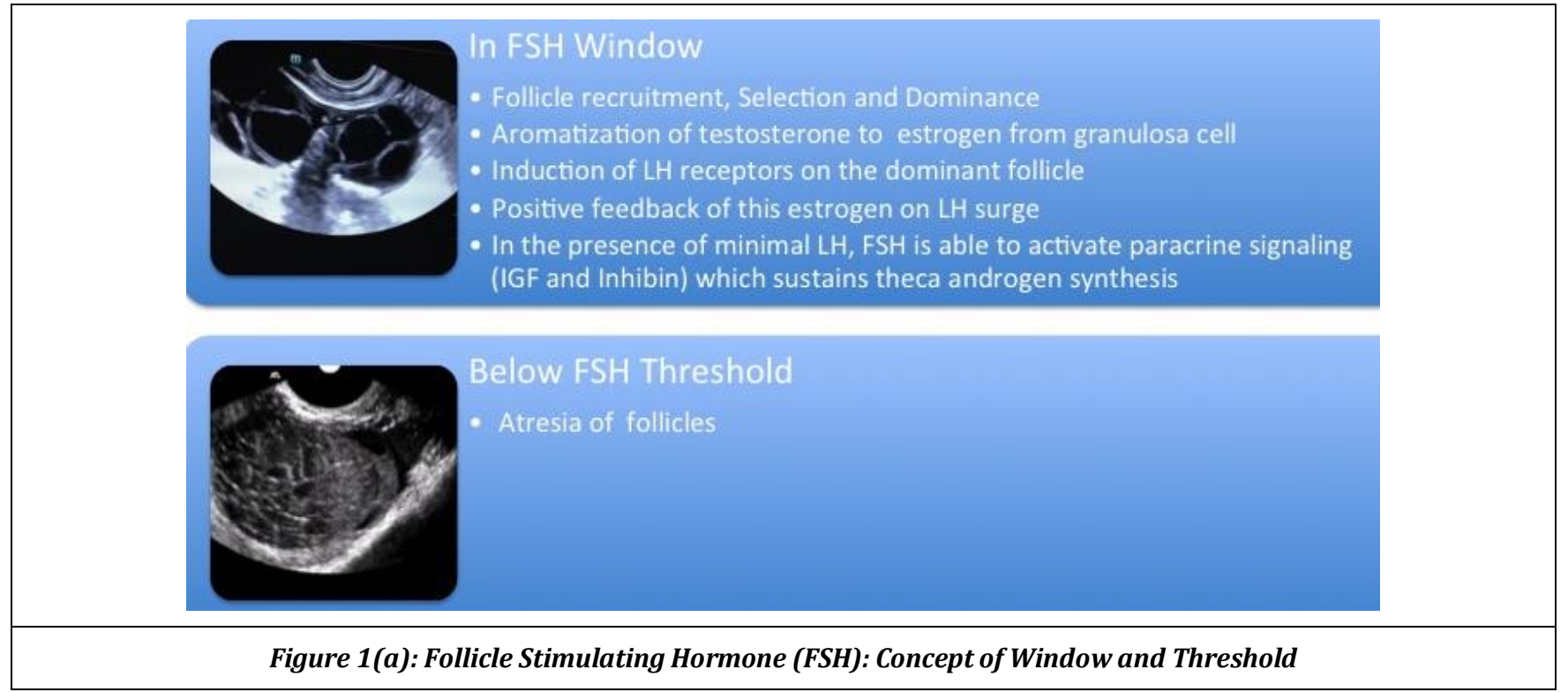

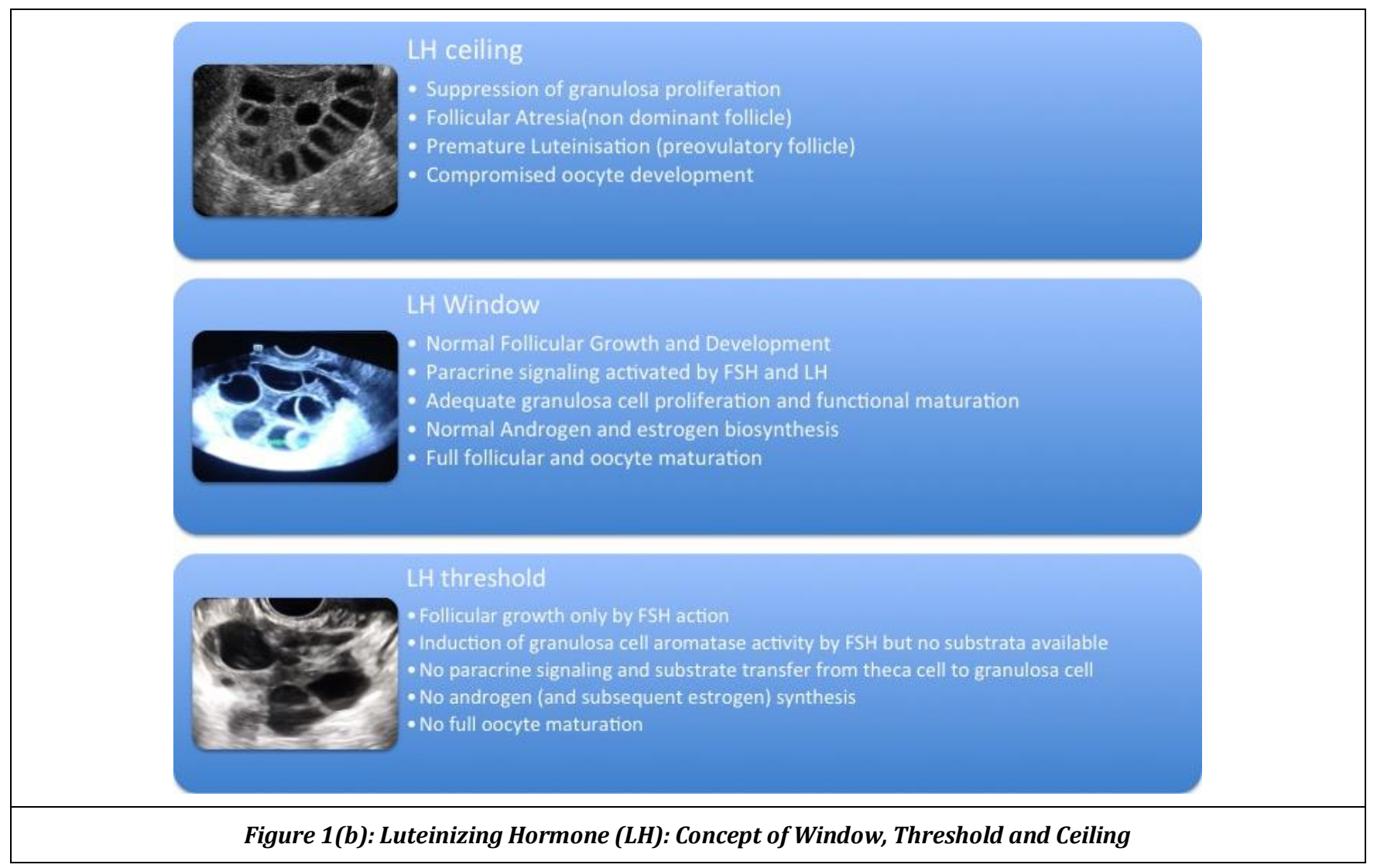

In a large study in Middle East the formula total dose of gonadotropins was given as 1.035X Age+2.355X FSH+

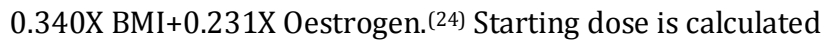
by dividing the total dose by 12 . Calculated starting dose is given for five days. On day 6 folliculometry scan is done to modify the dose. With this dose regimen $60 \%$ women had satisfactory response and $40 \%$ had their dose modulated. The dose was reduced for $25 \%$ women and the dose was increased for $15 \%$ women.

\section{Dose Protocols}

Gonadotropins have been used in different protocols. The step-up protocol, step- down protocol, chronic low dose protocol and the sequential step-up. The step-down protocol is most physiological as it mimics the physiological plateau and fall of FSH with rising levels of estradiol (negative feedback loop). The step-down protocol applies decremented doses of Gonadotropins once ovarian response is established.

Starting dose is higher to approximate physiological circumstances. The advantage of step-down protocol is decreased risk of Ovarian Hyper stimulation Syndrome. Step-down with low dose protocol is ideal for polycystic ovaries. 


\begin{tabular}{|l|l|l|l|}
\hline Age & $<21 \mathrm{BMI}$ & $21-25 \mathrm{BMI}$ & $>25 \mathrm{BMI}$ \\
\hline $21-25$ Years & $75 \mathrm{IU}$ & $150 \mathrm{IU}$ & $225 \mathrm{IU}$ \\
\hline $26-30$ Years & $150 \mathrm{IU}$ & $225 \mathrm{IU}$ & $300 \mathrm{IU}$ \\
\hline $31-35$ Years & $225 \mathrm{IU}$ & $300 \mathrm{IU}$ & $375 \mathrm{IU}$ \\
\hline $35-40$ Years & $300 \mathrm{IU}$ & $375 \mathrm{IU}$ & $450 \mathrm{IU}$ \\
\hline
\end{tabular}

Table 1. Starting Dose of Follicle Stimulating Hormone (FSH) Based on Age and Body Mass Index (BMI)

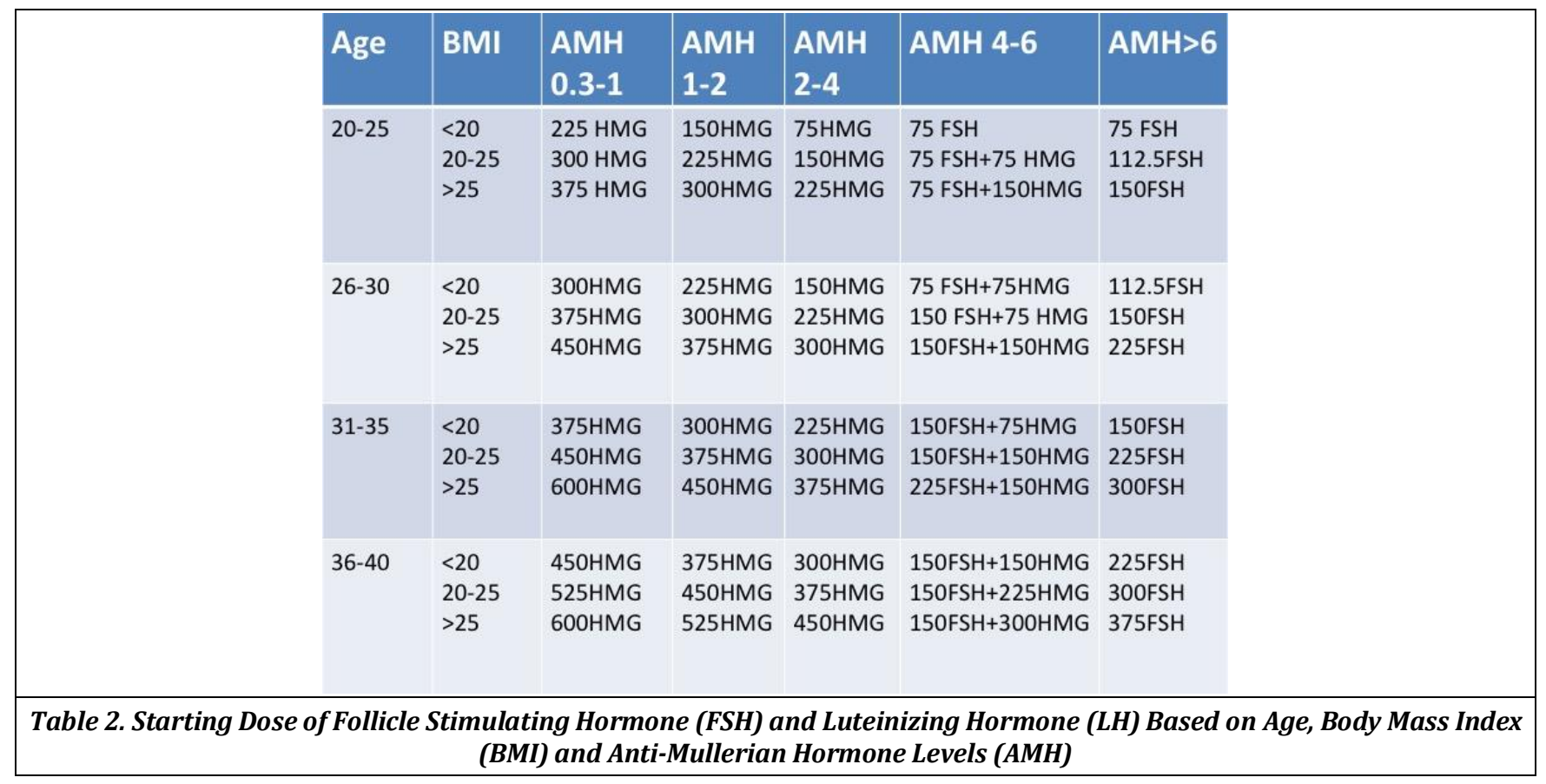

\section{Rise of Progesterone in the Follicular Phase}

There is a small but significant rise in the production of progesterone. Facilitates positive feedback response of oestrogen on LH. Induces second FSH surge ensuring completion of FSH action in the follicle. However, a premature progesterone rise in stimulated cycles is detrimental. High progesterone levels in follicular phase may lead to premature LH surge, premature luteinisation (Luteinized Unruptured Follicle). This impairs quality of oocyte and it is mandatory to check progesterone levels at beginning of cycle $(<0.9 \mathrm{ng} / \mathrm{l})$.

\section{How much FSH and HMG as Starting Dose? Based on Age and BMI}

The starting dose of Gonadotropins can be calculated depending on Age and BMI of patients. In normal BMI (2125 ), for ages between 20-25 years two ampules (150 IU FSH) is required, between 26-30 years three ampules (225 IU FSH) is required, between 31-35 years four ampules (300 IU FSH) is required and for ages between $36-40$ years five ampules (375 IU FSH) is required. Add one ampule for BMI $>25$ and subtract one ampule for $\mathrm{BMI}<21$. The age and BMI calibration of dose is given in Table 1.

\section{Based on FSH: LH Ratio}

The starting dose of FSH and HMG depends on FSH to LH ratio. If Ratio of FSH to $\mathrm{LH}$ is more than 2, equal amounts of FSH and HMG can be given. If FSH to LH ratio is less than 1, all FSH should be given (FSH: $\mathrm{LH}$ is $<1$ in polycystic ovaries). If the ratio of FSH to $\mathrm{LH}$ is between 1 and 2, 2/3 FSH and 1/3 HMG can be given.

\section{Based on AMH Levels}

If serum AMH levels are more than $6 \mathrm{ng} / \mathrm{ml}$, recombinant or urinary FSH can be used. If serum AMH levels are $4-6 \mathrm{ng} / \mathrm{ml}$, it will be prudent to use 2/3 FSH and 1/3 HMG. If Serum AMH levels are $2-4 \mathrm{ng} / \mathrm{ml}$, equal $\mathrm{FSH}$ and $\mathrm{HMG}$ can be used. If Serum AMH levels are $1-2 \mathrm{ng} / \mathrm{ml}$ it is wise to use only HMG. If serum AMH levels are below $1 \mathrm{ng} / \mathrm{ml}$ high doses of HMG 450$600 \mathrm{IU}$ are required. Table 2 shows the different starting doses in patients according to age, BMI serum AMH values. It is important to appreciate that follicular response to gonadotropins is titrated on a logarithmic scale and more concentration of gonadotropins are required to fill the binding sites as the binding sites get occupied. An extra $75 \mathrm{IU}$ FSH may be used discretely for patients with history of endometriosis, previous myomectomy and pelvic adhesiolysis.

\section{CONCLUSIONS}

Increase in FSH receptor content leads to Induction of $\mathrm{LH}$ receptors on the theca cells (action is enhanced by autocrine \& paracrine factors). Androgen substrate is essential for Aromatization of androgens to oestrogen under the influence of FSH.

LH is essential in folliculogenesis. It has been postulated to be the missing link when an IVF cycle result is unsatisfactory. LH is involved in selection \& growth and maturation of dominant follicle. $\mathrm{LH}$ is also responsible for the atresia of smaller follicles. LH is essential for ovulation and maintenance of corpus luteum.

To conclude, in the follicular phase of the cycle, FSH plays a crucial part in recruitment, selection and dominance. $\mathrm{LH}$ 
contributes to dominance, final maturation and ovulation. As there is no uniform clinical, biochemical, biophysical profile of patients, there is similarly no ideal controlled ovarian hyper stimulation protocol that is suitable for every patient.

The Dose depends on the following factors-

1. Age, BMI.

2. Ovulatory function and dysfunction.

3. Ovarian reserve as measured by antimullerian hormone and basal FSH levels.

4. Hormone profile (Serum testosterone, estradiol).

5. Associated Endometriosis, fibroids, previous pelvic surgeries.

6. Previous response to fertility drugs.

\section{REFERENCES}

[1] Speroff L, Fritz MA. Clinical gynecologic endocrinology and infertility. Wolters Kluwer 2013.

[2] Inge GB, Brinsden PR, Elder KT. Oocyte number per live birth in IVF: were Edward and Steptoe less wasteful? Hum Reprod 2005;20(3):588-92.

[3] Ferraretti AP, La Marca A, Fauser BC, et al. ESHRE consensus on the definition of 'poor response' to ovarian stimulation for in vitro fertilization: the Bologna criteria. Hum Reprod 2011;26(7):1616-24.

[4] Ahemmed B, Sundarapandian V, Gutgutia R, et al. Outcomes and recommendations of an Indian expert panel for improved practice in controlled ovarian stimulation for assisted reproductive technology. Int J Reprod Med 2017;2017:9451235.

[5] Lemmen JG, Rodríguez NM, Andreasen LD, et al. The total pregnancy potential per oocyte aspiration after assisted reproduction-in how many cycles are biologically competent oocytes available? J Assist Reprod Genet 2016;33(7):849-54.

[6] Daya S. Updated meta-analysis of recombinant folliclestimulating hormone (FSH) versus urinary FSH for ovarian stimulation in assisted reproduction. Fertil Steril 2002;77(4):711-4.

[7] Daya S, Ledger W, Auray JP, et al. Cost-effectiveness modelling of recombinant FSH versus urinary FSH in assisted reproduction techniques in the UK. Hum Reprod 2001;16(12):2563-9.

[8] Al-Inany H, Aboulghar M, Mansour R, et al. Meta-analysis of recombinant versus urinary-derived FSH: an update. Hum Reprod 2003;18(2):305-13.

[9] Andersen AN, Devroey P, Arce JC. A randomized trial (MERIT) comparing highly purified menotrophin and recombinant FSH in IVF. Hum Reprod 2005;20(suppl. 1):0-054.

[10] Balasch J, Barri PN. Reflections on the costeffectiveness of recombinant FSH in assisted reproduction. The clinician's perspective. J Assist Reprod Genet 2001;18(2):45-55.
[11] Bancsi LF, Broekmans FJ, Eijkemans MJ, et al. Predictors of poor ovarian response in in vitro fertilization: prospective study basal markers of ovarian reserve. Fertil Steril 2002;77(2):328-36.

[12] Kim HH. Markers of ovarian reserve: is it possible to estimate an ovarian age? Fertil Steril 2017;108(6):950-1.

[13] Park HJ, Lee GH, Gong du S, et al. The meaning of antiMüllerian hormone levels in patients at a high risk of poor ovarian response. Clin Exp Reprod Med 2016;43(3):139-45.

[14] Kunt C, Ozaksit G, Keskin Kurt R, et al. Anti-Mullerian hormone is a better marker than inhibin $\mathrm{B}$, follicle stimulating hormone, estradiol or antral follicle count in predicting the outcome of in vitro fertilization. Arch Gynecol Obstet 2011;283(6):1415-21.

[15] La Marca A, Grisendi V, Giulini S, et al. Individualization of the FSH starting dose in IVF/ICSI cycles using the antral follicle count. J Ovarian Res 2013;6(1):11.

[16] Fauser BC. Follicle pool depletion: factors involved and implications. Fertil Steril 2000;74(4):629-30.

[17] Pinborg A, Gaarslev C, Hougaard CO, et al. Influence of female bodyweight on IVF outcome: a longitudinal multicenter cohort study of 487 infertile couples. Reprod Biomed Online 2011;23(4):490-9.

[18] Abdalla H, Thum MY. An elevated basal FSH reflects a quantitative rather than qualitative decline of the ovarian reserve. Hum Reprod 2004;19(4):893-8.

[19] Panchal S, Nagori C. Ultrasound-based decision making on stimulation protocol for super ovulated intrauterine insemination cycles. International Journal of Infertility and Fetal Medicine 2016;7(1):7-13.

[20] Kupesic S, Kurjak A. Predictors of IVF outcome by three-dimensional ultrasound. Hum Reprod 2002;17(4):950-5.

[21] de Castro EC, de Freitas Borges AL, de Rezende KN, et al. Antral follicle count in predicting appropriate dose of gonadotropin in in vitro fertilization cycles. Reprod Clim 2014;29(3):136-42.

[22] Popovic-Todorovic B, Loft A, Lindhard A, et al. A prospective study of predictive factors of ovarian response in 'standard' IVF/ICSI patients treated with recombinant FSH. A suggestion for a recombinant FSH dosage normogram. Hum Reprod 2003;18(4):781-7.

[23] Olivennes F, Howles CM, Borini A, et al. Individualizing FSH dose for assisted reproduction using a novel algorithm: the CONSORT study. Reprod Biomed Online 2009;18(2):195-204.

[24] Hashish NM, Shaeer EK. Choosing the optimal dose of human menopausal gonadotropins for ovarian stimulation in ICSI cycle. Middle East Fertility Society Journal 2014;19(2):124-8. 